Umweltschutz und Beschäftigung

\section{Eine Debatte mit Tradition}

\author{
Schon Ende der achtziger Jahre wurde die Frage nach den \\ Beschäftigungswirkungen des Umweltschutzes als ein \\ „Evergreen“ der wirtschaftspolitischen Debatte bezeichnet. \\ Warum wird heute (immer) noch über diesen Zusammenhang \\ diskutiert und teilweise auch gestritten? Von Dietmar Edler
}

\begin{abstract}
D ie Schaffung und Sicherung von Arbeitsplätzen und eine Verbesserung des Umweltzustandes sind nach wie vor breit akzeptierte und hochrangige wirtschafts- und gesellschaftspolitische Ziele. Es darf deshalb nicht überraschen, dass aus politischer Sicht eine Verknüpfung beider Zielbereiche verlockend ist und somit Strategien, die ökologische Problembereiche in Angriff nehmen und gleichzeitig zur Lösung von Ungleichgewichten auf dem Arbeitsmarkt beitragen, hoch im Kurs stehen. Ein Fokus auf mögliche Synergien von Umweltpolitik und Wirtschaftspolitik ist selbst dann sinnvoll, wenn Umweltschutz richtigerweise als eine eigenständige politische Aufgabe mit eigenen Qualitätszielen verstanden wird.
\end{abstract}

\section{Ein Blick zurück}

Im Rückblick auf die Debatte zum Thema Umweltschutz und Beschäftigung werden sowohl sich wiederholende Argumentationsmuster wie auch deutliche Akzentverschiebungen erkennbar. In den siebziger Jahren - ziemlich bald nach Beginn einer systematischen Umweltpolitik in Deutschland - wurden erstmalig die Kostenbelastungen durch den Umweltschutz stärker thematisiert. Bezeichnenderweise geschah dies zu einem Zeitpunkt, als es durch den Ölpreisschock zu Rezession und steigender Arbeitslosigkeit kam. Themen wie umweltschutzbedingter Investitionsstau, Nachteile im internationalen (Kosten-)Wettbewerb und Standortverlagerungen wurden erstmals angesprochen. Aus dieser Zeit stammen die Schlagworte vom Umweltschutz als Jobkiller beziehungsweise als Jobknüller. In den achtziger Jahren war - unterstützt durch empirisch fundierte Untersuchungen zu den tatsächlichen Beschäftigungseffekten der Umweltpolitik - eine zunehmende Versachlichung der Debatte zu beobachten. Erste Versuche einer Integration von Umwelt- und Beschäftigungspolitik führten zur Konzipierung beschäftigungsfördernder Konjunkturund Wachstumsprogramme mit einem Fokus auf Umweltschutzinvestitionen wie das so genannte Zukunftsinvestitionsprogramm. Am Anfang der neunziger Jahre beherrschte zunächst die ökologische Aufbaupolitik für die neuen Bundesländer die Diskussion. Von der ökologischen Sanierung und vom nachholenden Aufbau der Umweltschutzinfrastruktur wurden erhebliche Impulse für den Arbeitsmarkt in den neuen Ländern erwartet, eine Erwartung, die sich nur teilweise erfüllte. Im Verlauf der neunziger Jahre wurde zum einen angesichts steigender Grenzvermeidungskosten stärker über eine Verschiebung vom nachgelagerten Umweltschutz (End-ofPipe) in Richtung integrierter Umweltschutz diskutiert. Der Nachweis der Beschäftigungswirkungen des integrierten Umweltschutzes erwies sich als methodisch komplex und als empirisch kaum leistbar. Zum anderen rückte jedoch ausgelöst durch die zunehmende Bedeutung des Klimaschutzes - die Ökosteuer in den Mittelpunkt des Interesses. Bei der
Begründung der Vorteilhaftigkeit wurde neben instrumentellen Effizienzüberlegungen explizit auf die möglichen Beschäftigungsgewinne durch eine Ökosteuer hingewiesen. Auch wenn die Spannweite der (modellmäßig) geschätzten Beschäftigungseffekte groß war, wird die ökologische Steuerreform als ein Erfolgsbeispiel einer Verknüpfung von ökologischen und ökonomischen Zielvorstellungen angesehen.

In den letzten Jahren wurde die Debatte um Umweltschutz und Beschäftigung zunehmend in den breiteren Kontext der nachhaltigen Entwicklung eingebettet. Da in Deutschland ein dreidimensionales Nachhaltigkeitsverständnis mit den Säulen ökologische, ökonomische und soziale Nachhaltigkeit in den Vordergrund gerückt ist, nicht zuletzt seit der Vorlage einer nationalen Nachhaltigkeitsstrategie durch die Bundesregierung, ist die Verknüpfung von Umweltschutz und wirtschaftlicher Entwicklung quasi institutionalisiert, denn zu einer dauerhaft nachhaltigen Entwicklung gehört auch ein hohes Beschäftigungsniveau.

\section{Umweltschutz ist eigener Politikbereich}

Es wurde schon betont, dass die Umweltpolitik als eigenständiger Politikbereich mit eigenen Zielen gesehen werden sollte. Letztlich zerstört unterlassener Umweltschutz langfristig die Wirtschaftsund die Lebensgrundlagen insgesamt. Dennoch ist es legitim, bei der Gestaltung von Umweltschutzmaßnahmen zu berücksichtigen, dass deren Umsetzung auch ökonomische und damit in der Regel Beschäftigungswirkungen auslöst. Auch wenn diese aus umweltpolitischer Sicht nur Nebeneffekte sind, ist es gleichwohl von Interesse, die Beschäftigungswirkungen der Umweltpolitik zu kennen. Durch diese Kenntnisse können mögliche Synergien aufgespürt und etwaige Konflikte verringert werden. Auch kann vermieden werden, dass falsche Vorstellungen über die tatsächlichen Beschäftigungseffekte der Umweltpolitik zum Hemmschuh für eine wirkungsvolle Umweltpolitik werden. 


\section{Brutto- und Netto- Beschäftigungseffekte}

Wie viele Menschen für den Umweltschutz in Deutschland arbeiten, ist vorrangig ein Problem der zuverlässigen empirischen Schätzung und damit in weit geringerem Maße kontrovers als die Frage, ob eine bestimmte Umweltmaßnahme oder ein bestimmtes umweltpolitisches Instrument per Saldo in der gesamten Volkswirtschaft mehr Arbeitsplätze schafft oder Arbeitsplätze kostet. Die erste Fragestellung nach den Bruttobeschäftigungseffekten lässt sich für Deutschland mit einem kombinierten angebots- und nachfrageorientierten Schätzansatz mittlerweile einigermaßen breit gefächert und zuverlässig beantworten. Auf sie wird unten etwas ausführlicher eingegangen.

Die zweite Fragestellung, also die nach einer Wirkungsanalyse des Umweltschutzes, erfordert den Einsatz von modellbasierten Analyseinstrumenten und wurde bisher kaum flächendeckend durchgeführt. Verbreitet sind solche Wirkungsanalysen jedoch für ausgewählte Instrumente des Umweltschutzes, etwa die Ökosteuer, oder für bestimmte umweltrelevante Themenfelder wie Erneuerbare Energien. Die Verallgemeinerung der aus solchen spezifischen Untersuchungen gewonnenen Ergebnisse ist natürlich nur mit großer Vorsicht möglich. Auf Basis einer Vielzahl solcher Studien lässt sich jedoch folgern, dass für eine Mehrheit der untersuchten Umweltschutzmaßnahmen sich positive Nettobeschäftigungseffekte ergeben, die allerdings in der Regel - vor allem im Vergleich zum Bruttobeschäftigungseffekt - eher gering ausfallen.

\section{Beschäftigung im Umweltschutz}

Betrachtet man die Brutto-Beschäftigungseffekte des Umweltschutzes, so wird deutlich, dass Umweltschutz mittlerweile eine bedeutende Quelle für Beschäftigung in Deutschland ist. Die letzte Schätzung kommt zu dem Ergebnis, dass im Jahr 2002 ungefähr 1,4 Millionen Menschen in Deutschland Beschäftigung hatten, weil ökonomische Ressourcen zur Beseitigung oder Vermeidung von Umweltproblemen eingesetzt werden. Das sind ungefähr 3,7 Prozent der gesamten Beschäftigung in der deutschen Volkswirtschaft. Die Beschäftigung im Umweltschutz folgt weitgehend Trends, die auch sonst in der Beschäftigungsentwicklung zu erkennen sind. Zum einen gibt es eine abnehmende Bedeutung der Güterproduktion und eine zunehmende Bedeutung von Dienstleistung auch im Umweltschutzbereich, und zum anderen haben wir die auch allgemein zutreffende Feststellung, dass besser qualifizierte Menschen bessere Chancen auf dem Arbeitsmarkt haben als schlechter qualifizierte.

Von den Umweltschutzbeschäftigten waren rund 960.000 unmittelbar mit Umweltschutzaktivitäten befasst, überwiegend in Dienstleistungsbereichen. Ungefähr 260.000 Personen hatten eine Beschäftigung, weil Umweltschutz-Investitionen getätigt wurden, rund 190.000 wurden für den Betrieb von bestehenden Umweltschutzanlagen benötigt, gut 45.000 Personen fanden Beschäftigung, weil in anderen Ländern deutsche Umweltgüter nachgefragt wurden. Hatte die Umweltschutzbeschäftigung in den neunziger Jahren noch zugenommen, so ist es in den „klassischen“ Umweltschutzbereichen nunmehr zu einer Stagnation beziehungsweise zu einem leichten Rückgang gekommen. Bei einem gegebenen hohen Niveau der Umweltschutzinfrastruktur fehlen außerhalb der Energiepolitik in der umweltpolitischen Gesetzgebung derzeit Initiativen, die nachfragestimulierende Effekte für die Umweltbeschäftigung entfalten könnten. Die Klimapolitik und speziell die Förderung der Erneuerbaren Energien war der Schwerpunktbereich der deutschen Umweltpolitik in den letzten Jahren - eine Entwicklung, die man eindeutig an der Beschäftigungsentwicklung im Umweltschutz ablesen kann. So ist die den Erneuerbaren Energien zurechenbare Beschäftigung von 66.600 Personen im Jahr 1998 auf knapp 120.000 Personen im Jahr 2002 gestiegen, was einem Zuwachs von nahezu 80 Prozent in vier Jahren ent- spricht. Besonders die Windkraft ist in diesem Zeitraum zum größten Beschäftigungsbereich aufgestiegen. Die Erneuerbaren Energien haben sich also zu einem dynamischen Wachstumsfeld der Umweltschutzbeschäftigung in Deutschland entwickelt.

\section{Ein knapper Blick voraus}

Die Ungleichgewichte auf dem Arbeitsmarkt und die Notwendigkeit weitere Aktivitäten zum Schutz der Umwelt werden die gesellschaftliche Entwicklung auf absehbare Zeit weiter begleiten. Damit spricht alles dafür, dass auch die Debatte um die Wechselwirkungen von Umweltschutz und Beschäftigung noch nicht abgeschlossen ist, sondern sich weiterentwickeln wird. Im Prinzip wird in der Nachhaltigkeitsstrategie der Regierung das positive Zusammenwirken von Umweltschutz und wirtschaftlicher Entwicklung gesehen und als Strategieelement anerkannt. Eine realistische Betrachtung des politischen Prozesses zeigt jedoch, dass derzeit andere wirtschaftspolitische Strategien und auch Themen im Vordergrund stehen. Die Chancen einer ökologischen Modernisierungsstrategie werden nur in Ausnahmefällen offensiv vertreten. Teilweise wird auch ein „Rollback“ der Umweltpolitik aus vermeintlich ökonomischen Gründen befürchtet.

Ein erstes Indiz könnte die weitere Entwicklung der Erneuerbaren Energien liefern. Hier wurde durch eine zielgerichtete Umweltpolitik ein Wachstumsmarkt mit spürbaren Beschäftigungseffekten in seiner Entstehung begünstigt. Es wurden Innovationen angestoßen und eine im internationalen Wettbewerb günstige Position mit Chancen für einen Lead-Markt geschaffen. Es wäre bedauerlich, wenn diese Entwicklung nicht fortgesetzt würde.

I AUTOR + KONTAKT

Dr. Dietmar Edler ist wissenschaftlicher Referent am Deutschen Institut für Wirtschaftsforschung (DIW).

DIW, Königin-Luise-Straße 5, 14195 Berlin. Tel.030/8 97 89-280, E-Mail: dedler@diw.de 
(c) 20I0 Authors; licensee IÖW and oekom verlag. This is an article distributed under the terms of the Creative Commons Attribution Non-Commercial No Derivates License (http://creativecommons.org/licenses/by-nc-nd/3.o/), which permits unrestricted use, distribution, and reproduction in any medium, provided the original work is properly cited. 